\title{
Nicotine Does Not Improve Discrimination of Brain Stimulation Reward by Rats
}

\author{
P. B. S. Clarke* and R. Kumar \\ Department of Psychiatry, Institute of Psychiatry, De Crespigny Park, London SE5 8AF, United Kingdom
}

\begin{abstract}
Rats were trained to shuttle between two selected ("ON") arms of a Y maze, to obtain electrical stimulation of the medial forebrain bundle. Each shuttle response was rewarded with a brief pulse train. Repetitive entries into the same "ON" arm were not rewarded, nor were entries made into the third ("OFF") arm. Every 67s, stimulation was made available from a different pair of arms. Test sessions lasted for $80 \mathrm{~min}$, beginning immediately after $\mathrm{SC}$ injection. Undrugged subjects responded faster, and with a greater proportion of rewarded responses, the higher the stimulation current.

In non-tolerant rats, nicotine $(0-0.4 \mathrm{mg} / \mathrm{kg})$ depressed responding and induced ataxia shortly after injection; from $40 \mathrm{~min}$, nicotine increased low rates of responding but decreased high rates. All these effects were dose-dependent. Mecamylamine $(2.0 \mathrm{mg} / \mathrm{kg})$ prevented the initial depressant action. With repeated daily injections of nicotine $(0.4 \mathrm{mg} / \mathrm{kg})$, a marked stimulant action emerged which replaced the initial depressant action, and this was dose-dependent. However, responding was increased by nicotine even when brain stimulation was not available ("time-out"). In contrast, an additional "rate-free" index based on discrimination showed that nicotine did not augment the rewarding properties of the brain stimulation.
\end{abstract}

Key words: Intracranial self-stimulation - Brain stimulation reward - Rate-free index - Nicotine - Mecamylamine Chronic administration - Tolerance - Abstinence - Rat

It is assumed that most people smoke tobacco in order to obtain nicotine, and recent studies suggest that laboratory animals can learn to self-administer this drug (Goldberg et al. 1981; Nelson and Cox 1982). However, it is not known how nicotine produces its rewarding effects.

In non-tolerant rats trained to press a lever to obtain rewarding electrical brain stimulation, nicotine depresses responding shortly after systemic injection of large doses, especially if the baseline rate of responding is high, whereas responding is generally stimulated at longer intervals after injection and if the baseline rate is low (Pradhan and Bowling 1971; Olds and Domino 1969a,b; Wanner and Bättig 1966). Similar principles apply over a range of behaviours, such as responding for food or water (Morrison 1967; Stitzer et al.

Offprint requests to: $\mathrm{P}$. B. S. Clarke

* Present address: Biological Psychiatry Branch, Building 10 Room 3N256, N.I.M.H., Bethesda, MD 20205, USA
1970), avoidance responding (Bignami and Michalek 1978), and locomotor activity (Morrison and Lee 1968; Clarke and Kumar 1982). It is therefore necessary to determine whether the drug alters the reward strength of the electrical stimulation per se; a drug may produce nonspecific alterations of responding either by acting independently of the electrical stimulation, or by interacting with a consequence of the electrical stimulation (such as motor disturbance) which affects responding without impinging on central reward processes. A procedure was developed in the present study which provided two indices of the rewarding properties of brain stimulation, with one of the measures being independent of the rate of responding. The first experiment consisted of a dose response study of the effects of nicotine on responding for brain stimulation reward in rats which had not previously received the drug.

The second experiment investigated whether effects of nicotine on ICSS could be prevented by mecamylamine. This secondary amine blocks the ganglionic stimulant actions of nicotine in the peripheral nervous system (Stone et al. 1956) and is thought to act centrally as well (Bennet et al. 1957). In most, if not all, studies so far reported the behavioural actions of nicotine have been prevented by this drug (e.g. Stitzer et al. 1970; Clarke and Kumar 1982).

Although tobacco smoking can be a life-long habit, the chronic behavioural actions of nicotine have received little attention. In one preliminary study (Pradhan and Bowling 1971) tolerance developed to the rate-depressant action of this drug in rats trained to press a lever in order to obtain rewarding electrical stimulation of the brain.

In the third experiment, the effects of nicotine on ICSS were studied over a 3-week period during which all subjects received a constant daily dose of nicotine. The initial ratedepressant action of the drug receded over this period; nicotine came increasingly to enhance rates of responding, but did not detectably increase the rate-independent measure of brain stimulation reward. This finding was then confirmed with a range of doses. Responding was then measured over several days of abstinence. Finally, subjects were tested for residual tolerance to the depressant effects of nicotine.

\section{Materials and Methods}

Subjects. Male hooded rats (Olac 76 Ltd., Bicester, UK), weighing between 290 and $350 \mathrm{~g}$ at surgery, were used; they were housed singly in a room illuminated from 8.00 to 20.00 hours, and had constant access to food and water. Subjects were handled daily for 1 week before surgery. 
Surgery. The rats were anaesthetised with sodium pentobarbitone and were each implanted with a bipolar insulated stainless steel electrode (Plastic Products Co., Roanoke, VA, USA, MS 303/3), using a stereotaxic apparatus (Stoelting Co., Chicago, IL, USA). Electrodes were aimed at the medial forebrain bundle at the level of the lateral hypothalamus, on the left side [De Groot (1959): A 5.4 L 1.8 V-2.8].

Histology. Each rat was sacrificed with an overdose of sodium pentobarbitone, and the electrode removed. The brain was fixed in $10 \%$ formol saline for several weeks. Coronal sections, $50 \mu \mathrm{m}$ thick, were taken and dried in paraformaldehyde vapour. Sections were then stained in $0.1 \%$ solutions of luxol fast blue and cresyl fast violet.

Apparatus. This consisted of two Y mazes painted black inside (arm length $21.5 \mathrm{~cm}$, arm width $15 \mathrm{~cm}$, height $53 \mathrm{~cm}$ ). Each lay on a formica sheet which was wiped clean between test sessions. A $12 \mathrm{~V}$ signal light was recessed in the end of each arm, $6 \mathrm{~cm}$ above the floor. All three lights were on whenever brain stimulation was available. Entries into individual arms were detected by interruptions of infra-red photobeams which ran across each arm $2.5 \mathrm{~cm}$ from its end, $3.3 \mathrm{~cm}$ above the floor.

Solid state equipment (BRS/LVE), situated in an adjacent room, was programmed to determine when electrical brain stimulation was available, and to reward the animal for an appropriate response. Brain stimulation was delivered via leads connected to a mercury commutator (BRS/LVE) clamped above the centre of the maze.

ICSS Procedure. Starting between 2 and 3 weeks after surgery, rats were trained to shuttle between two selected arms of the maze (termed "ON" arms) in order to obtain brain stimulation. A single pulse train was delivered when the rat interrupted the photobeam in an "ON" arm, provided that the preceding response had been made in a different arm. Hence, the rat was required to shuttle back and forth between the two "ON" arms. The remaining "OFF" arm did not provide brain stimulation, but it monitored unrewarded responses. Rewarded and unrewarded responses were registered separately. Only shuttle responses were registered; repetitive entries into the same "ON" or "OFF" arm were not recorded.

In the early training sessions, the "ON" arms were not changed until responding had been reliably established. They were then reselected with gradually increasing frequency, until the final experimental conditions were reached. At this stage, each session lasted $80 \mathrm{~min}$ and was subdivided into a series of $67 \mathrm{~s}$ units grouped in threes, during the first two of which brain stimulation was available at a given current level and the third consisted of "time out", when brain stimulation was not available at all and the lights at the ends of the three arms were switched off.

At the start of each 67-s unit in which current was available, a different pair of "ON" arms was selected. After each sequence of three 67-s units, a new current intensity became available. Each of six current levels was provided once in each quarter of the session.

The brain stimulation consisted of biphasic pulse pairs, timed electronically (Digitimer) as follows: train duration $200 \mathrm{~ms}$; pulse pair frequency $100 \mathrm{~Hz}$; pulse width $0.5 \mathrm{~ms}$; inter-pulse interval (onset to onset) $5 \mathrm{~ms}$. Current intensities were in equal logarithmic steps $(25-141 \mu \mathrm{A})$, preset on a current stepping device coupled with constant current stimulus isolators (Digitimer), and calibrated as the voltage drop across a $1-\mathrm{Kohm}$ resistor.

Pilot experiments confirmed that the rats made most unrewarded responses just after a new pair of "ON" arms had been selected, and they then quickly settled down to make a high proportion of rewarded responses. Accordingly, the accuracy of discrimination was defined as the proportion of rewarded responses out of the first ten which were made after the "ON" arms were changed each time. Repetitive entries were not counted. The measure of accuracy was determined at each current intensity. As long as at least ten responses were made within each period of $67 \mathrm{~s}$, the accuracy of discrimination provided a rate-free measure of brain stimulation reward. Undirected responding resulted in a score of around $66.6 \%$

Drugs. (-)-Nicotine $\mathrm{H}(+)$ tartrate (BDH) was dissolved in physiological saline and neutralised to $\mathrm{pH} 7.2 \pm 0.2$ with $\mathrm{NaOH}$. Mecamylamine $\mathrm{HCl}$ (Merck) and hexamethonium bromide (Sigma) were also dissolved in saline. Injections were subcutaneous, made into the flank in a volume of $1 \mathrm{ml} / \mathrm{kg}$. Subjects were tested for $80 \mathrm{~min}$, starting immediately after injection of nicotine or saline (control). Doses refer to the base of the compound.

Analysis of Data. Multivariate analysis of variance was used, each rat serving as its own control. Accuracy of discrimination scores were given an arcsin square root transformation, as suggested by Winer (1970, p 221), to stabilise the variances. Data were analysed for trends across absolute values of current and dose. A "dose-dependent" effect refers to a significant linear trend. Specific comparisons between control (i.e. saline) and other levels of dose refer to paired $t$-tests, and probability values are 2 -tailed.

\section{Experiment 1}

Effects of Nicotine on ICSS before Chronic Treatment. After training, 11 rats which responded at stable rates for brain stimulation were chosen for tests with nicotine. Tests were 3 or 4 days apart, and each subject received each dose of nicotine $(0,0.025,0.05,0.1,0.2,0.4 \mathrm{mg} / \mathrm{kg})$ in a random order. In addition, one saline test preceded and another followed the block of drug testing. A 40-min "warm up" session directly preceded each injection. Stimulation current was made available in a random order which was changed between successive tests.

\section{Experiment 2}

Effects of Nicotine on ICSS in Non-Tolerant Rats Following pretreatment with Mecamylamine. One month later, eight of the subjects were retrained for tests with mecamylamine. The warm up session was omitted, and a pretreatment injection of mecamylamine $(0,2 \mathrm{mg} / \mathrm{kg})$ was given $20 \mathrm{~min}$ before the treatment injection of nicotine $(0,0.4 \mathrm{mg} / \mathrm{kg})$. Each rat received each of the four drug combinations once, in a Williams square design (Cox 1958).

To simplify programming, two pseudo-random orders of current were used. Each rat received two sessions with each current order, and the two current orders occurred equally frequently in each drug condition. 

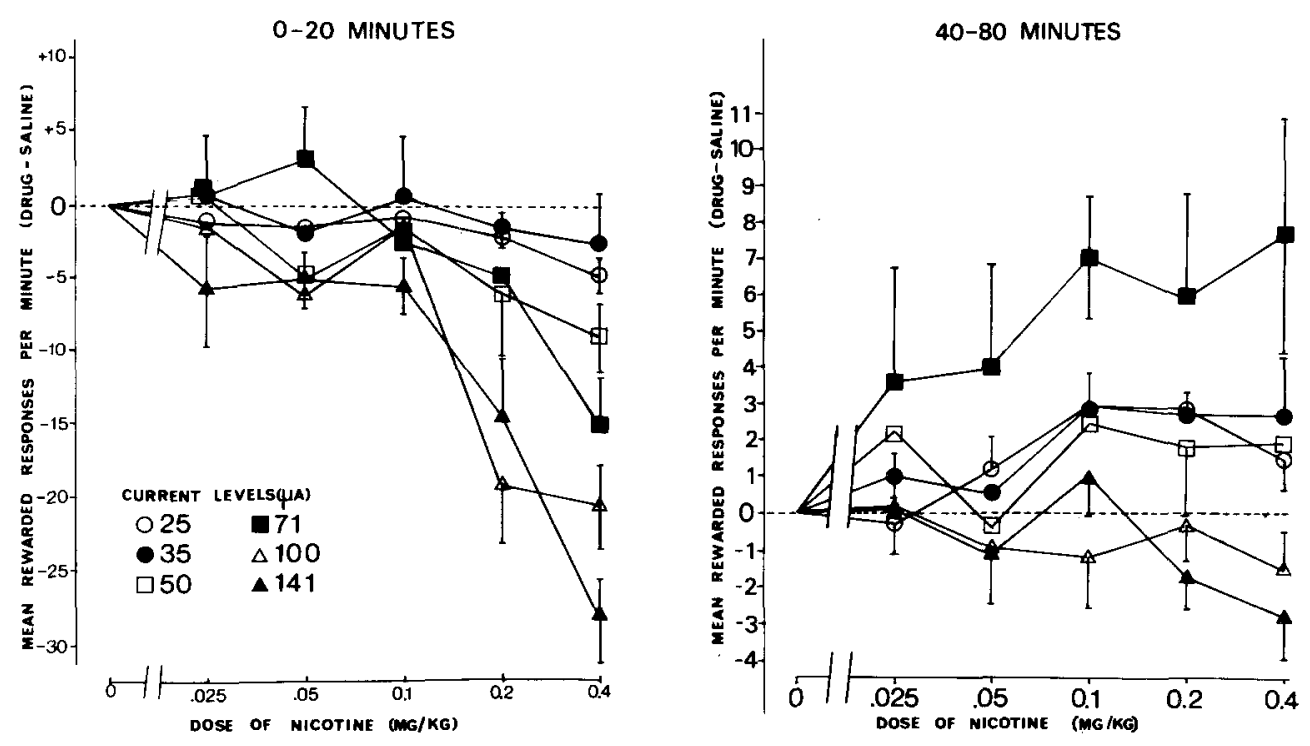

Fig. 1

The effects of nicotine on rate of rewarded responding. Rats $(n=11)$, initially drug naive, were tested twice weekly with each dose of SC nicotine. This figure shows the mean difference $( \pm$ SEM) of scores between the nicotine tests and the saline test. In the first $20 \mathrm{~min}$ after injection (left graph), responding was reduced in a dose-related way. From 40 to 80 min (right graph), responding was significantly increased at 25 and $71 \mu \mathrm{A}$, but fast responding at the highest current was depressed

\section{Experiment 3}

Effects of Nicotine on ICSS in Rats Maintained on a Constant Daily Dose of the Drug. Seven of the eight remaining subjects were used; they now weighed $424-516 \mathrm{~g}$. One rat lost its implant and was excluded from day 19 onwards.

Stable responding was re-established and daily testing was then started. After four saline tests (days 1-4), subjects were tested alternately with nicotine $(0.4 \mathrm{mg} / \mathrm{kg})$ and saline (days 5-18). Three rats were tested with nicotine on odd-numbered days and the other four on even-numbered days. Each subject was maintained on a constant daily dose of $0.4 \mathrm{mg} / \mathrm{kg}$, following its first nicotine test. On saline test days, the maintenance injection was given immediately after the session. Rats tested with $0.4 \mathrm{mg} / \mathrm{kg}$ of nicotine received no further injection on that day.

From days 19-24, a dose-response study was carried out in the six remaining subjects. As before, nicotine test days alternated with saline test days, and subjects continued to received a total daily dose of $0.4 \mathrm{mg} / \mathrm{kg}$. Each rat was tested once with each dose of nicotine $(0.005,0.1,0.2 \mathrm{mg} / \mathrm{kg})$ in a Latin square (Cox 1958) with two subjects per group.

Subjects then remained in their home cages for 1 week, receiving nicotine $0.4 \mathrm{mg} / \mathrm{kg}$ daily. Two tests with nicotine $(0.4 \mathrm{mg} / \mathrm{kg})$ followed, on days 33 and 34 , and chronic injectons were discontinued. The rats were then tested with saline during 3 weeks of abstinence from nicotine, on days $35-38,41,44,47,49,54$ and 55. Finally, each subject was tested with nicotine $(0.4 \mathrm{mg} / \mathrm{kg})$ on day 56 to assess residual tolerance.

As in experiment 2, two pseudo-random current orders were used. These were counterbalanced across subjects and across drug treatments.

\section{Results}

\section{Experiment 1}

\section{Effects of Nicotine on ICSS Before Chronic Treatment}

Tests with Saline. Neither the rate of rewarded responding nor the accuracy of discrimination varied across the three saline tests, at any current level $(P>0.05$ for each test), and responding in time-out was also stable. Rewarded responding and accuracy both increased with increasing current (linear trends, respectively: $F=167.3, \quad P<0.0001 ; \quad F=158.9$, $P<0.0001, d f 1,10)$. Responding was faster in the first $20 \mathrm{~min}$ than in the rest of the session (rewarded $F=19.7, d f 1,10$, $P<0.005$; time-out $F=10.7, d f 1,10, P<0.01$ ).

Test with Nicotine. Preliminary analyses showed that the effects of nicotine (linear trend over dose) changed across successive quarters of the 80-min test session, and accordingly the first two quarters were analysed separately. In the first 20 min, nicotine markedly depressed both rewarded and unrewarded responding in a dose-related way $(F=122.6$, df $1,10, p<0.001 ; F=26.0$, df $1,10, \quad P<0.0005$, respectively; Fig. 1). Rewarded responding was most reduced at high current, whereas low rates of responding, whether at low current or in time-out (Fig. 6), was less affected. Nicotine also produced ataxia and prostration, especially at the higher doses.

From 20 to $40 \mathrm{~min}$, responding was no longer profoundly depressed. Nicotine reduced rewarded responding in a dosedependent way only at the two highest currents. Time-out responding was actually stimulated at certain doses (Fig. 6).

From 40 to $80 \mathrm{~min}$, the actions of nicotine were predominantly stimulant, although a dose-dependent depression of rewarded responding could still be detected at the highest current $(F=9.0$, df 1,10, $P<0.02)$. Current intensity was clearly important in determining the effect of nicotine on the rate of rewarded responding; this is shown by a significant interaction between the linear components of trend on dose and current $(F=13.9$, df $1,10, P<0.005)$. Rewarded responding was increased in a dose-related way at 25 and $71 \mu \mathrm{A}$ $(P<0.02$ in either case - Fig. 1). Nicotine also increased time-out responding in a dose-related manner $(F=26.8$, df 1,10, $P<0.0005$; Fig. 6).

The accuracy of discrimination could not be measured in the first $20 \mathrm{~min}$ after injection, because few responses were made under the drug. From 40 to $80 \mathrm{~min}$, despite the changes in response rate, accuracy was not significantly altered by the drug; trends on dose at individual current levels were all nonsignificant. 


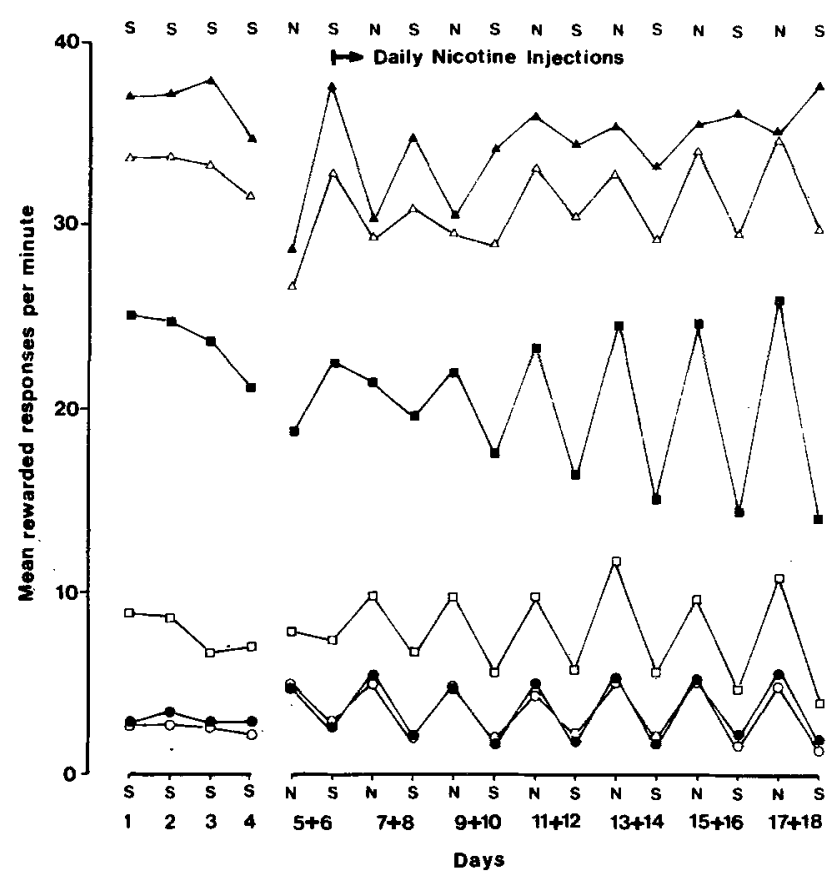

Experiment 2

Effects of Nicotine on ICSS in Non-Tolerant Rats Following Pretreatment with Mecamylamine

When given alone, mecamylamine reduced the rate of rewarded responding $(F=42.1, d f 1,7, P<0.0005)$. This effect did not vary across the session, and was independent of current intensity. Time-out responding was also reduced $(F=8.2, d f 1,7, P<0.05)$.

As in experiment 1 , nicotine alone significantly depressed rewarded responding and time-out responding in the first 20 min. Mecamylamine completely prevented these actions, and there was a significant interaction between the effects of the two drugs $(F=35.4, d f 1,7, P<0.001 ; F=5.9, d f 1,7$, $P<0.05)$. From 40 to $80 \mathrm{~min}$, nicotine given alone stimulated time-out responding $(P<0.05)$; rewarded responding was increased at the three lowest current levels $(P<0.05$ for each), but depressed at the highest current $(P<0.05)$. In the presence of mecamylamine, these effects were not statistically significant, but on neither measure was there a significant interaction between the two drugs. Both nicotine and mecamylamine given alone tended to impair the accuracy of discrimination from 40 to $80 \mathrm{~min}$, but a significant effect did not occur at any current intensity; nevertheless, at the two highest currents, there was an interaction between the two drugs $(P<0.05)$, suggesting that their actions tended to cancel out.

\section{Experiment 3}

Effects of Nicotine on ICSS in Rats Maintained on a Constant Daily Dose of the Drug

Saline Baseline (Days 1-4). Rewarded responding and the accuracy of discrimination were stable across the four consecutive saline tests, at each current level $(P>0.05$ for

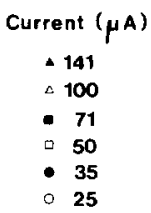

Fig. 2

The effects of chronic nicotine on rates of rewarded responding. After four saline test sessions, rats ( $n=7$ ) were tested daily, alternately just before or just after an injection of nicotine $(0.4 \mathrm{mg} / \mathrm{kg} \mathrm{SC})$. At first (days 5 and 6$)$, nicotine enhanced low rates of responding and depressed high rates. Over subsequent days, the depressant action waned and a stimulant action emerged. Baseline (non-drug) response rates declined over successive tests

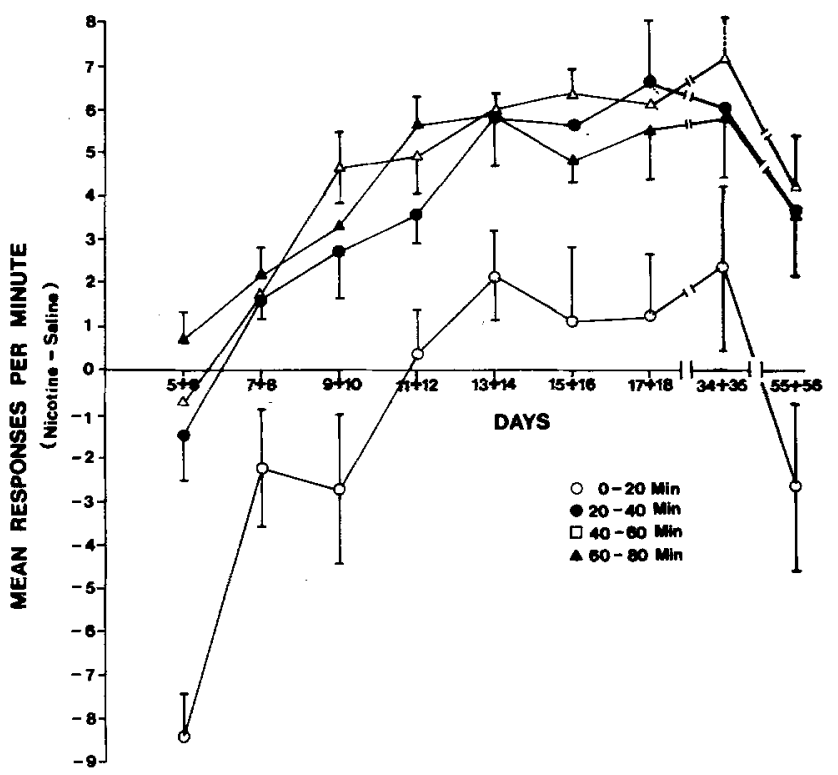

Fig. 3. The effects of chronic nicotine on rates of rewarded responding. The vertical axis shows the mean ( \pm SEM) difference between nicotine and saline tests, of rewarded responses in the four quarters of the session $(n=6$ or 7 ). Whilst subjects received nicotine $0.4 \mathrm{mg} / \mathrm{kg} \mathrm{SC}$ every day (days $6-35$ ), nicotine came increasingly to stimulate responding and the depressant action was lost. Tolerance to the depressant action persisted in abstinence (days 55 and 56)

each comparison; Fig. 2). However, responding in time-out did vary between days $(F=64.9$, df $3,4, P<0.001)$.

Daily Injections of Nicotine (days 5-18). Rewarded responding declined over successive saline tests, except at the highest current level (linear trend over days $F=6.2, d f 1,6, P<0.05$ for each; Fig. 2), whereas accuracy did not change significantly.

This phase was subdivided into seven consecutive pairs of days, each consisting of one saline test and one nicotine test 

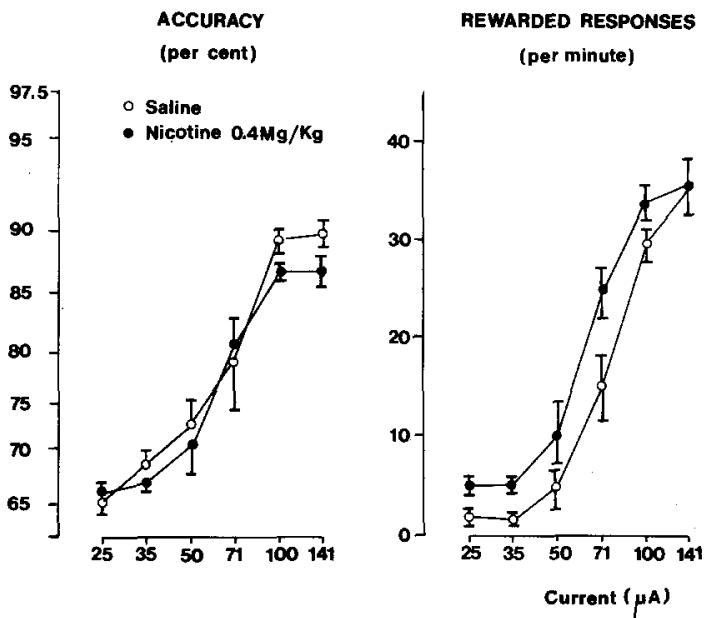

Fig. 4. The effects of nicotine on accuracy and rates of rewarded responding in tolerant rats (days $11-18$ ). Accuracy data are plotted as the mean ( \pm SEM) of the transformed individual scores $(n=7)$, in equal aresin square root units. Nicotine $(0.4 \mathrm{mg} / \mathrm{kg} \mathrm{SC})$ significantly enhanced rates of rewarded responding without significantly increasing accuracy

for each animal. The drug effect was defined as the difference between the nicotine and saline scores within each pair of tests.

In the first $20 \mathrm{~min}$ rewarded responding was at first markedly reduced, but over successive tests, this depressant action disappeared, and a slight overall stimulant action emerged (Fig. 3). Drug-induced ataxia also waned during this period, but remained detectable.

Between 20 and 80 min after injection, a stimulant action was evident from the second nicotine test onward (Fig. 3). This increased over days of testing (linear trend of drug effect over days $F>25.7, d f 1,6, P<0.005$ for each session quarter).

Over the whole session, the emergent stimulant action of nicotine was associated not only with a decline of rewarded responding under saline (see above), but also with an increase in rates of rewarded responding over successive nicotine tests $(50,100,141 \mu \mathrm{A}: P<0.01$ for each). As before, the depressant effect of nicotine on rewarded responding was most persistent at the highest current level. Taking the 2-week period as a whole, nicotine increased time-out responding $(F=110.2, d f 1,6, P<0.0001)$. This stimulant effect increased over successive days $(F=15.8, d f 1,6, P<0.01)$, and as in the case of rewarded responding, the greatest change of drug effect occurred in the first $20 \mathrm{~min}$ after injection.

Was accuracy improved by nicotine under conditions where rewarded responding was increased? From days 11 to 18 , nicotine produced little or no depressant action, and significantly increased rewarded responding at every current level except the highest $(F=7.87$ to $F=77.4, d f 1,6, P<0.05$ to $P<0.005$; Fig. 4). In contrast, accuracy was impaired at $100 \mu \mathrm{A}(F=7.87$, df $1,6, P<0.05)$, and otherwise unaltered (Fig. 4).

Dose-Response Study in Tolerant Rats. The analysis of data included not only the counterbalanced block of tests with nicotine $(0-0.2 \mathrm{mg} / \mathrm{kg})$ on days $19-24$, but also the saline and nicotine $(0.4 \mathrm{mg} / \mathrm{kg})$ tests on days 17 and 18 ; the order of drug presentation was treated as random.

After preliminary analysis, the data from all four saline test sessions were pooled. Over the whole 80 -min session,

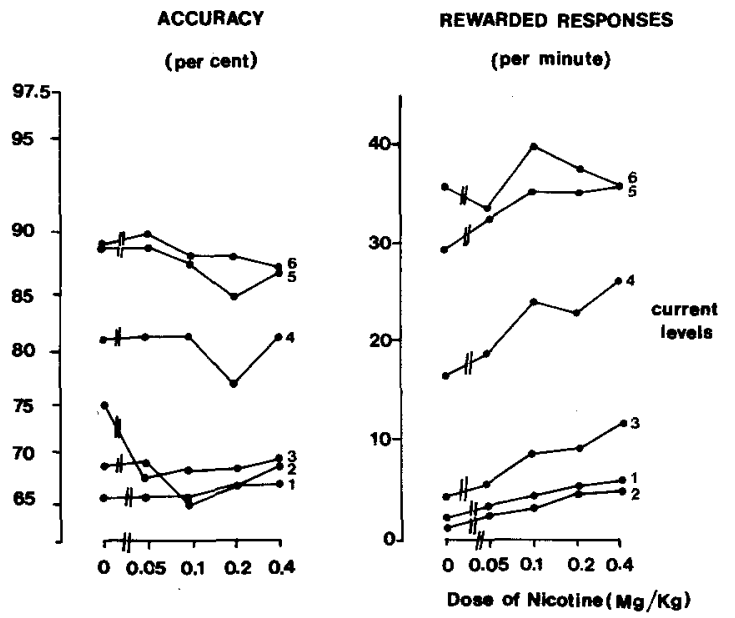

Fig. 5. Dose-response study in tolerant rats. Accuracy data are plotted as the mean ( $\pm \mathrm{SEM})$ of the transformed individual scores $(n=6)$, in equal arcsin square root units. Current levels $1-6$ refer to current intensities of $25-141 \mu \mathrm{A}$ in ascending order. Nicotine increased rates of rewarded responding in a dose-related way $(25-70.7 \mu \mathrm{A})$, without significantly altering accuracy

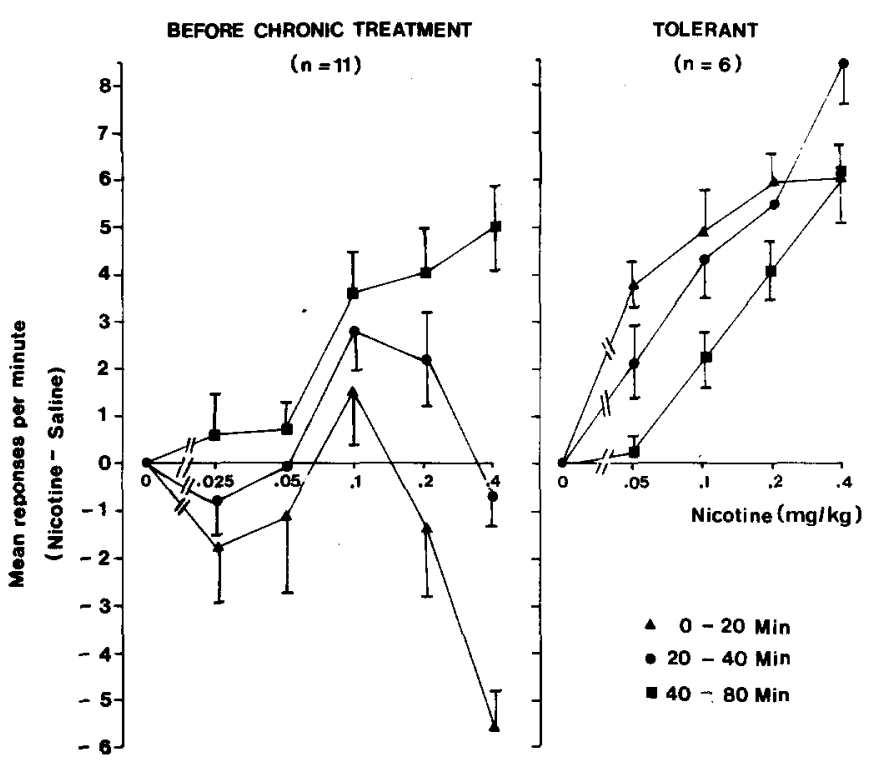

Fig. 6. Responding in the absence of brain stimulation ("time-out"). The vertical axis represents the mean difference ( \pm SEM) of scores between nicotine and saline tests. Before chronic treatment, nicotine increased responding in "time-out", but only after an initial period of depression. After repeated injections of nicotine, the depressant action was replaced by a dose-related stimulant action which occurred throughout the $80-\mathrm{min}$ session

nicotine enhanced rewarded responding in a dose-related way at the four lowest current levels $(F=12.15$ to $46.42, d f 1,5$, $P<0.05$ to $P<0.005 ;$ Fig. 5), and responding in time-out was also stimulated $(F=291.4, d f 1,5, P<0.0001$; Fig. 6$)$. Even in the first $20 \mathrm{~min}$, low rates of responding were significantly increased by the drug, both at the two lowest current intensities and in time-out (Fig. 6). Nicotine increased the rate of rewarded responding without affecting the accuracy of discrimination. This is demonstrated by the lack of any significant linear trend over dose $(F<3.42$, df 1,5 , $P>0.1$ at every current intensity; Fig. 6). However, since 
accuracy scores at a given current varied considerably between subjects, a "threshold" value of current was assigned to each rat, which under saline conditions resulted in an accuracy score closest to $75 \%$. Analysis of trends over dose, using data from non-tolerant and tolerant rats, failed to show any significant effects of nicotine on accuracy.

Abstinence (Days 35-55). When daily nicotine injections were discontinued, responding in time-out rose over successive saline tests $(F=18.7$, df 1,5, $P<0.01)$. A similar but non-significant trend occurred in the rate of rewarded responding. Neither measure changed significantly over the four consecutive daily tests in abstinence.

Residual Tolerance. Nicotine depressed rewarded responding $(0-20 \mathrm{~min})$ to a greater extent before the start of chronic injections than on days 55 and $56(F=7.65$, df $1,5, P<0.05$; Fig. 3). Thus, some tolerance persisted after 3 weeks of abstinence. A similar comparison was made between days 34 and 35, just before chronic injections were stopped, and days 55 and 56. This indicated that some tolerance had been lost ( $F=8.28, d f 1,5, P<0.05$; Fig. 3).

Histology. Microscopic examination identified six electrode sites in the medial forebrain bundle/lateral hypothalamic region, three sites in the overlying zona incerta, and one site just dorsal to the zona incerta. One electrode placement could not be located.

\section{Discussion}

This study confirms that in non-tolerant rats, nicotine can depress or stimulate responding, depending on the dose, time after injection, and on the undrugged response rate. In tolerant rats however, nicotine increased overall response rates without detectably enhancing brain stimulation reward; to our knowledge, these are new findings.

An initial depression of responding following systemic injection of nicotine has been reported to occur with a similar time course in a variety of behavioural tests (Morrison 1967; Stitzer et al. 1970; Domino and Lutz 1973) including studies of ICSS (Pradhan and Bowling 1971; Wanner and Bättig 1966), and it may therefore represent a general disruption of behaviour. In the present study, the rats became flaccid and ataxic within about $2 \mathrm{~min}$ of injection and subsequently became prostrated with the transient loss of righting reflex at the two highest doses. These symptoms wore off between 5 and $15 \mathrm{~min}$ after injection, and were seen in other rats given the drug in the home cage. Comparable injections of nicotine in anaesthetised rats produced a marked depression of spinal reflexes (Stephenson and Clarke, in preparation).

After the initial depressant effects of nicotine had subsided, rates of responding were enhanced at low and intermediate levels of current, whereas responding remained slightly reduced at the highest current. A persistent reduction of responding at high control rates has also been seen with water reward (Stitzer et al, 1970). These observations are consistent with previous studies (see introduction) suggesting that nicotine exerts rate-dependent effects on responding in non-tolerant rats. As the session progressed, the baseline rates of responding declined by only a small amount, except at low current levels. Thus the emergence of a stimulant action cannot be explained solely by a constant rate-dependent effect occurring against a background of falling control rates of responding. A similar conclusion can be drawn from studies of operant behaviour (Morrison 1967).

Nicotine strongly enhanced responding in periods of timeout in the second half of the session, and this action resembled that seen at low levels of current. Although time-out was signalled, the subjects did not withhold responses when reward was not available, and so it is unclear whether timeout responding reflects unconditioned locomotor activity or responding in anticipation of brain stimulation. Nicotine has also been reported to increase responding in time-out in rats trained to lever press for food or to avoid shock (Pradhan 1970).

In the second experiment, mecamylamine clearly blocked the depressant actions of nicotine on the rate of responding; ataxia was also prevented. These findings are consistent with studies of locomotor activity (Clarke and Kumar 1982), of operant responding (Stitzer et al. 1970; Spealman et al. 1981) and of ICSS (Olds and Domino 1969a). There was also the suggestion that the stimulant actions of nicotine were reduced or blocked by mecamylamine, but the evidence is equivocal in part because nicotine when given alone exerted only a weak stimulant action.

At the single dose used, mecamylamine depressed responding for brain stimulation and also in time-out. This drug did not visibly alter the appearance or behaviour of the animals, and there was little evidence that under mecamylamine, the brain stimulation was less rewarding.

With repeated exposure to nicotine, the depressant action rapidly disappeared; response rates returned to non-drug baseline levels or rose above them, and signs of motor impairment also receded. The drug action became predominantly stimulant, and this was shown to be dose-related after 2 weeks of chronic treatment.

The experimental design in the third experiment did not incorporate a control group injected daily with saline instead of nicotine; therefore, the possibility cannot be excluded that the changing response to nicotine occurred as a result of repeated testing or some other process associated with the passage of time. In particular, it is not possible to ascertain whether the decline in response rates (though not of accuracy) across successive saline sessions, when the rats were tested before their daily nicotine injection, reflected the gradual emergence of acute signs of withdrawal. This might indicate an increasing dependence on the drug, but such a decline could have happened in the absence of maintenance injections, through a passive process such as tissue damage at the site of electrical stimulation. When daily nicotine injections were stopped, response rates tended to recover towards pre-drug levels; this argues against the occurrence of some sort of irreversible change such as tissue damage, but may be attributed to the adoption of spaced test sessions during the abstinence phase.

In tolerant rats, nicotine markedly increased not only rewarded responding but also responding when brain stimulation was not available. These actions strongly resemble the reported effects of this drug on unconditioned motor activity, where with daily administration, a persistent form of tolerance developed to the initial depression and a stimulant action emerged (Morrison and Stephenson 1972; Clarke and Kumar 1982). Tolerance to a depressant action has also been seen in rats lever-pressing for water reward (Domino and Lutz 1973). In the present study, tolerance did not develop to the stimulant action of nicotine, and parallel findings have been 
obtained from investigations of locomotor activity (Kuschinksy and Hotovy 1943; Morrison and Stephenson 1972; Clarke and Kumar 1982).

One drawback of the procedure used here is that the accuracy measure cannot be reliably ascertained when response rates are low. Thus, it could not be determined whether the great reduction in responding seen after large doses of nicotine in non-tolerant animals was due to an alteration of brain stimulation reward as well as to a motor deficit. However, in both non-tolerant and tolerant rats, the drug increased rates of responding without increasing the accuracy of discrimination. This suggests that nicotine did not enhance the reward strength of electrical stimulation of the medial forebrain bundle, although it exerted a behavioural stimulant action. Nicotine thus appears to differ from certain other stimulant drugs which are selfadministered, notably amphetamine and cocaine, which are reported to enhance brain stimulation reward (Kornetsky et al. 1979).

Acknowledgements. We thank Mr. F. Goldsmith for building the currentstepping device, and Merck and Co. for a gift of mecamylamine. This work was supported by a Medical Research Council studentship awarded to P.B.S.C.

\section{References}

Bennet G, Tyler C, Zaimis E (1957) Mecamylamine and its mode of action. Lancet 2, 218-222

Bignami G, Michalek H (1978) Cholinergic mechanisms and aversively motivated behaviours. Ch. 4 In: Anisman H, Bignami G (eds) Psychopharmacology of aversively motivated behaviour. Plenum, New York, pp $173-256$

Clarke PBS, Kumar R (1982) The effects of nicotine on locomotor activity in non-tolerant and tolerant rats. $\mathrm{Br} \mathrm{J}$ Pharmacol (in press)

Cox DR (1958) Planning of experiments. Wiley, London

De Groot J (1959) The rat forebrain in stereotactic coordinates. Trans R Neth Acad Sci 52:No. 4

Domino EF, Lutz MP (1973) Tolerance to the effects of daily nicotine on rat bar pressing behaviour for water reinforcement. Pharmacol Biochem Behav 1:445-448
Goldberg SR, Spealman RD, Goldberg DM (1981) Persistent high-rate behaviour maintained by intravenous self-administration of nicotine. Science 214 (4250):573 - 575

Kornetsky C, Esposito RU, McLean S, Jacobson JO (1979) Intracranial self-stimulation thresholds - a model for the hedonic effects of drugs of abuse. Arch Gen Psychiatry 36:289-292

Kuschinksy G, Hotovy R (1943) Über die zentral erregende Wirkung des Nicotins. Klin Wschr 22:649-650

Morrison CF (1967) Effects of nicotine on operant behaviour of rats. Int J Neuropharmacol 6:229-240

Morrison CF, Lee PN (1968) A comparison of the effects of nicotine and physostigmine on a measure of activity in the rat. Psychopharmacology $13: 210-221$

Morrison CF, Stephenson JA (1972) The occurrence of tolerance to a central depressant effect of nicotine. Br J Pharmacol 46:151-156

Nelson WT, Cox B (1982) Nicotine self-administration in rat. Pharmacol Biochem Behav 15:833

Olds J, Domino EF (1969a) Comparison of muscarinic and nicotinic cholinergic agonists on self-stimulation behaviour. J Pharmacol Exp Ther 166:189-204

Olds J, Domino EF (1969b) Differential effects of cholinergic agonists on intracranial self-stimulation and escape behaviour. J Pharmacol Exp Ther 170:157-167

Pradhan SN (1970) Effects of nicotine on several schedules of behaviour in rats. Arch Int Pharmacodyn Ther 183:127-138

Pradhan SN, Bowling C (1971) Effects of nicotine on self-stimulation in rats. J Pharmacol Exp Ther 176:229 - 243

Spealman RD, Goldberg SR, Gardner ML (1981) Behavioural effects of nicotine. I. Schedule-controlled responding by squirrel monkeys. J Pharmacol Exp Ther 216:484-491

Stitzer M, Morrison J, Domino EF (1970) Effects of nicotine on fixed interval behaviour and modification by cholinergic antagonists. J Pharmacol Exp Ther 171:165-177

Stone CA, Torchiana ML, Navarro A, Beyer KH (1956) Ganglionic blocking properties of 3-methylaminoisocamphane hydrochloride (mecamylamine): A secondary amine. J Pharmacol Exp Ther $117: 169-183$

Wanner HU, Bättig K (1966) Wirkung von Nikotin und Amphetamin auf die Selbstreizung bei der Ratte. Helv Physiol Pharmacol Acta 24: $\mathrm{C} 122-124$

Winer BJ (1971) Statistical principles in experimental design: Second edition. McGraw-Hill, New York

Received August 7, 1982 